\title{
Some confounding factors in the study of preparatory set in reaction time*
}

\author{
ROGERS ELLIOTT \\ Dartmouth College, Hanover, New Hampshire 03755
}

\begin{abstract}
In Experiment 1, the effects of the range of the distribution of preparatory intervals (PIs) on the effect of PI on RT was demonstrated, and disparate conclusions about the development of preparatory set were thereby accounted for to some degree. In Experiment II, both range of the PIs and their average value were examined in a factorial design for their effects on RT, and it was concluded that, for any average value, greater range will result in greater PI effects; and for any range, greater average values will result in smaller PI effects on RT.
\end{abstract}

In a recent study (Elliott, 1970) of age differences in simple reaction time (RT), it was shown that when a number of different preparatory intervals (PIs-the time from a warning signal to a reaction signal) are presented randomly in a series of trials, the effect of PI on RT was much greater among young children than it was among adults. The form of the RT by PI function, called the preparatory set effect, was familiar as the one usually resulting from random presentations of members of a set of PIs of the values used $(1,2,4,8$, and $16 \mathrm{sec})$ : The shortest PI resulted in the longest RTs, the median PI produced the quickest RTs, and the longest $\mathrm{Pl}$ resulted in long RTs, so that the function produced was U shaped.

The form of the function is usually accounted for as follows. For the shortest PI of a set like the one used, it is especially difficult to be ready to respond for two reasons: The contingent probability of its occurrence, given that a reaction signal has not yet occurred, is the lowest among all the PIs; further, since the shortest PI is usually preceded by a longer PI, adaptation level effects work especially strongly against one's being ready for it. The deficit that appears with the longest PI only appears when that PI is both absolutely and relatively so long that a $S$ finds it difficult to maintain a readiness to respond: If it is not much longer than the other members in the set, RTs may be very quick to the longest PI, but there seems to be no circumstance in which they will be quick to the shortest one, given a random presentation of the PIs.

In the author's 1970 paper, the young children produced steeper (larger) preparatory set effects than the adults, and this fact was interpreted to be in keeping with data showing that such effects were also greater in other groups that are slower than normal young adults: e.g., the aged, the retarded, and the psychotic. Persons in these groups presumably neither get ready to react as quickly, nor maintain a readiness to react as long. The problem is that the present author had earlier reported

*These studies were conducted with support from Grants HD04047. HD01571, and HD12648 from the National Institute of Child Health and Human Development. The assistance of Sara Black and Richard Lyczak is very gratefully acknowledged.
(1964) that young children showed preparatory set effects that were slightly smaller than those produced by adults. He had argued at the time that the children might have been so inattentive as to be impervious to both adaptation level effects and contingent probabilities of occurrence of the PIs.

Among the possible reconciliations of these disparate results is one that depends on the fact that the parameters of the distributions of PI values used in the two designs were different. That is, the earlier study may have failed to show any age differences in the effects of PI values because there may have been little effect of PI to begin with. In the earlier study, the PIs ranged from about 3.2 to $4.7 \mathrm{sec}$ in rectangular distribution. It is possible that, given the central value of $4 \mathrm{sec}$, the range of about $1.5 \mathrm{sec}$ was so small that the different PIs were relatively indiscriminable one from the other. The PIs in a set would have to be discriminable to have an effect. and the discriminability of the values of a set would depend not only upon their range but also upon the length of their central PI (in a rectangular distribution of PIs). For example, in a set of five PIs, a range of $2 \mathrm{sec}$ might be indiscriminable where the PIs ranged from 10 to $12 \mathrm{sec}$ but quite discriminable where the PIs ranged from 1 to $3 \mathrm{sec}$.

The hypothesis, therefore, is that for any given central median value of a set of PIs, the effect of PIs on RT is a direct function of the range of the PIs and, for any range, an inverse function of the central value. Drazin (1961) has some data supporting the first part of this proposition, and Experiment II reports a supporting demonstration experiment in which both central value and size of range are varied factorially. But the problem of reconciling the disparate results of the author's two studies of age differences in RT was investigated first.

\section{EXPERIMENT I}

The major features of the design of this experiment were that the young $S s$ were from the same population (for the most part they were the same Ss) as those who had produced the comparatively large effects of PI on RT in the 1970 study. This time. though. they were to 
perform under a PI distribution much like that used in the 1964 study, where PI effects had been relatively small. In addition, they were run under a second PI distribution, roughly comparable to those used by Goodenough (1935) and Jones (1937) in their developmental studies of RT, both to extend the range of observation of PI effects and to assess the representativeness of the present sample.

\section{Method}

The Ss were 108 children, 12 (6 boys and 6 girls) at each year from 5 through 13, and 36 male and female adults. Except for the adults and the 5-year olds, all had served a year previous in an earlier RT study. The apparatus consisted of five interval timers, a stepping switch, an electronic counter, an audio-oscillator, earphones, and a microswitch that served as the response key.

The task was simple auditory RT, each trial being initiated by the S's pressing his response key, thereby starting the delay period (PI), which ended with the onset of the reaction signal, a 1000 -cycle tone of about $75 \mathrm{~dB}$. The reaction was a keylift, and the RT was displayed in milliseconds on a counter visible only to E.

The two PI distributions used in the study were the 4-sec set, with PIs of 3.0, 3.5, 4.0, 4.5, and $5.0 \mathrm{sec}$; and the 2-sec set, with PIs of $1.4,1.7,2.0,2.3$, and $2.6 \mathrm{sec}$. Each $\mathrm{S}$ was tested on the two different PI distributions twice, once in each half of the session, with counterbalancing of order within Ss and between the $\mathrm{Ss}$ in any age-condition-sex subgroup. The presentation of the PIs in a given distribution was programmed in a quasirandom sequence onto a 44-step stepping switch connected to the timers controlling the PIs. A complete cycle of the stepper produced four of the PIs nine times each, and the other eight times. For each distribution, 52 trials, or just over a cycle, were used, the first ones being considered as warm-up trials. The intertrial interval was simply the time required for the $E$ to record the $R T$, deliver noncontingent approval (under low incentive) or contingent reinforcement (under high incentive), and tell the $\mathrm{S}$ he could go ahead.

The incentive conditions were defined by the same sequences of high- and low-incentive patterns that had been used in the 1970 study: low-high, high-low, and low-low. For purposes of administering the incentives, each half of the session was treated as a unit, so that in the low-high condition, for example, both PI sets in the first half would be under low incentive, followed by a $5-\mathrm{min}$ rest, followed by the second half done under high incentive.

In the low-incentive condition, Ss were given a reward (money) for participating at the beginning of the half-session. The $\mathrm{E}$ encouraged the $\mathrm{S}$ to do as well as he could at the beginning of the trials and at the midpoint of the series. No knowledge of results was given, nor were any rewards contingent on quality of performance. Instead, the E said "good" or "fine," or otherwise indicated noncontingent satisfaction at the end of each reaction as he recorded it.

High-incentive conditions differed from low-incentive conditions in two ways: The reward was greater, and its attainment was contingent to some degree upon the speed of each reaction. The $E$ would covertly estimate from the practice trials or the previous series the average RT for his $S$. The $S$ was told that each time he did especially well he would be given money but that, when his performance was too slow, he would not win. The $E$ used the estimated average RT as his criterion for reward and attempted a payoff rate of about $50 \%$, covertly changing the criterial RT as necessary. The $S$ was never told what exact score was required or what score he had attained on any trial. Use of this incentive manipulation is detailed elsewhere (Elliott, 1966).
The nature of the incentives varied with the ages and followed a pattern in which a flat fee was given in low incentive and a smaller flat fee plus the money won contingently was given in high incentive. The payoffs for a low-incentive half-session were as follows: 5- and 6-year olds, $\$ .35 ; 7$ - and 8-year olds, $\$ .45 ; 9$ and 10-year olds, $\$ .55$. Under high incentive the flat fee payoffs were as follows: 5- and 6-year olds, $\$ .25 ; 7$ - and 8-year olds, $\$ .30$; 9- and 10 -year olds, $\$ .40$. For all ages in high incentive, the payoff rate included $\$ .01$ per satisfactory $R T$ in addition to the flat fee.

Since they were naive with respect to the task, the new 5-year olds were given a short separate preliminary session so that they would be to some degree habituated to and practiced at the data-taking session, as were the other children. The new adults were given a relatively long warmup at the data-taking session.

As in the 1970 study, ages were combined among the children in groups of three years: $5+6+7$, called sixes; $8+9+10$, called nines; $11+12+13$, called twelves; and adults. Analyses were of the same sort used in the 1970 paper, i.e., repeated measures, groups by trials, and repeated measures on more than one factor. Medians of the final nine RTs of each PI in any. PI set constituted the scores of each $S$ in the simple analyses of PI effects. There were, thus, five scores from each of the PI sets in each half-session.

\section{Results and Discussion}

Figures 1 and 2 illustrate the RTs for the various age groups, conditions, and sessions for the 2-sec set and the 4-sec set, respectively. In general, the size of the effect of PI was smaller among these Ss than it had been in the 1970 study, where the range of PIs had been much greater (from 1 to $16 \mathrm{sec}$ ). In the younger age groups of the 1970 study, differences in RT from the shortest $(1 \mathrm{sec})$ to the median $(4 \mathrm{sec}) \mathrm{Pl}$ were commonly $50-60 \mathrm{msec}$ and as much as $20 \mathrm{msec}$ in the older groups. In the present study, as can be seen, they were very much reduced. That fact, plus the smaller $\mathrm{N}$ of 12 in each group, meant that fewer of the RT by PI functions were statistically significant when assessed by repeated-measures analyses of variance with $d f=4 / 44$. The 2-sec set had slightly more reliable effects upon RT than did the 4-sec set, but in neither case was the Pl effect impressive, having been reliable $(p<.05)$ in a fashion not systematically related to the other independent variables of age, condition, or sessions in half of the 24 RT by PI functions in the 2-sec set and a quarter of them in the 4-sec set. [In a study employing two comparable series, Karlin (1959) also found that there was somewhat less reliability of the effect of $\mathrm{PI}$ in the longer of the two series.] By contrast, in the 1970 study using a PI set of much larger range, all but 1 of the 24 RT by PI functions were reliable.

Another way to compare the effects of the PI set of large range of the 1970 study with smaller ranged sets used here is to ask whether the functions produced by the latter are significantly flatter than those produced by the former. The comparison is especially pertinent when it pairs the 1970 set with the present 4-sec set, because those are the sets, approximately, involved in the disparity of results in the 1964 and 1970 experiments. The appropriate analysis of variance term to assess the 
Fig. 1. Means of the individual median

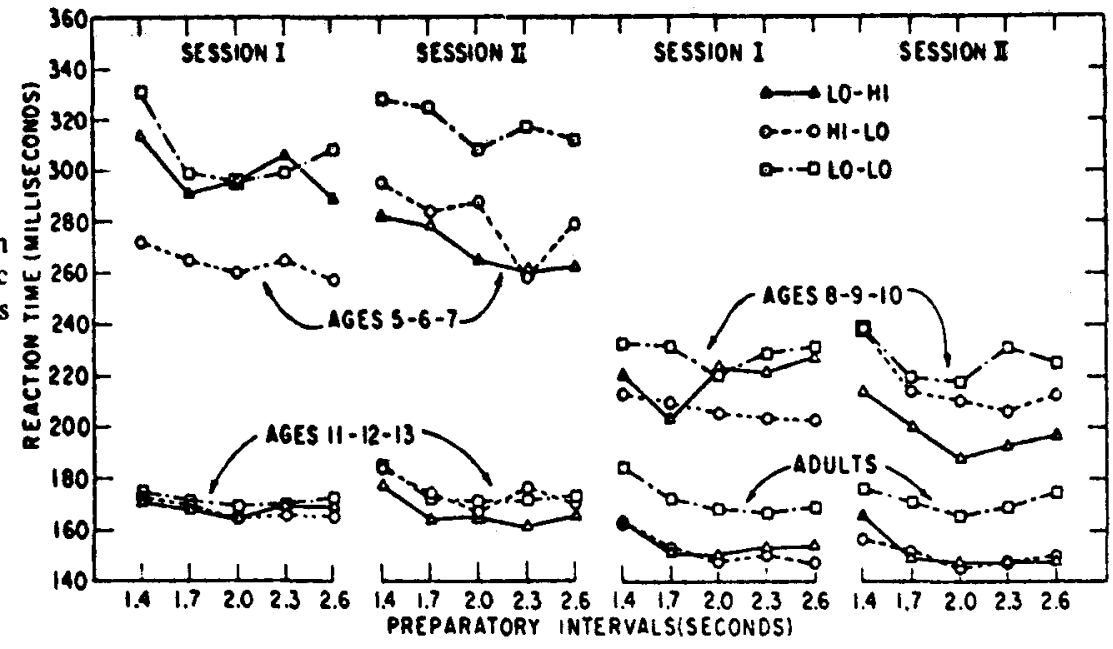

matter would be the interaction of PI set (large or small) with PI effect. But most of the children in the present study had also served in the 1970 study, so that the change in PI set was confounded with an increase in practice. That is, the first half of the present session amounts to the third session for most of these children.

There are data from another investigation, however, (Elliott, 1972) of a number of boys never previously tested on RT, who have had at least three sessions under the large (1970) PI set and under various incentive patterns, requiring at least three sessions. Matching for age, practice, and incentive history yielded two independent groups of 33 boys each, 10-years old or younger. In the analysis of variance, the data for the group under the 4-sec set was compared with that for the group under the large 1970 set. The Groups by Trials $F$ ratio was $3.43(d f=4 / 256)$, significant at less than the .025 level. The average RTs for the five PIs $(1,2,4,8$, and $16 \mathrm{sec}$ ) of the larger set were (in $\mathrm{msec}$ ) 345,311 , 308,331 , and 329 , respectively, roughly $U$ shaped and not so flat as the functions produced under the 4-sec set.

A similar analysis was conducted comparing the data from the 2-sec set of PIs with that from the 1970 set, with a similar result. The $F$ ratio for Groups by Trials interaction was 2.53, $\mathrm{df}=4 / 256, \mathrm{p}<.05$. Both of the ?and 4-sec sets yielded faster RTs than the large, 1970 sets (Fs of 21.7 and 12.7, respectively, $\mathrm{df}=1 / 64$, $\mathrm{p}<.001)$ : The averages, taken over conditions and PIs, were 258,273 , and $322 \mathrm{msec}$, respectively.

The adults in Experiment I were not the same as those in the 1970 paper, so that the first session data from the 1970 study were compared with the present first half-session data for the 2- and 4-sec sets. For the comparison, data of every other $S$ of the $72 \mathrm{Ss}$ in the 1970 study was selected to provide a group of 36 like the present one. In this case, neither interaction term was significant, probably because the RT by PI functions were relatively flat even in the large set. But, as with the boys, the RTs were faster with the shorter sets: The F ratios for the comparison of the 2- and $4 \cdot \mathrm{sec}$ set data
Fig. 2. Means of the individual median RTs at each PI, by age groups. for the 4-sec set. Each of the three incentive conditions is $z$ shown separately.

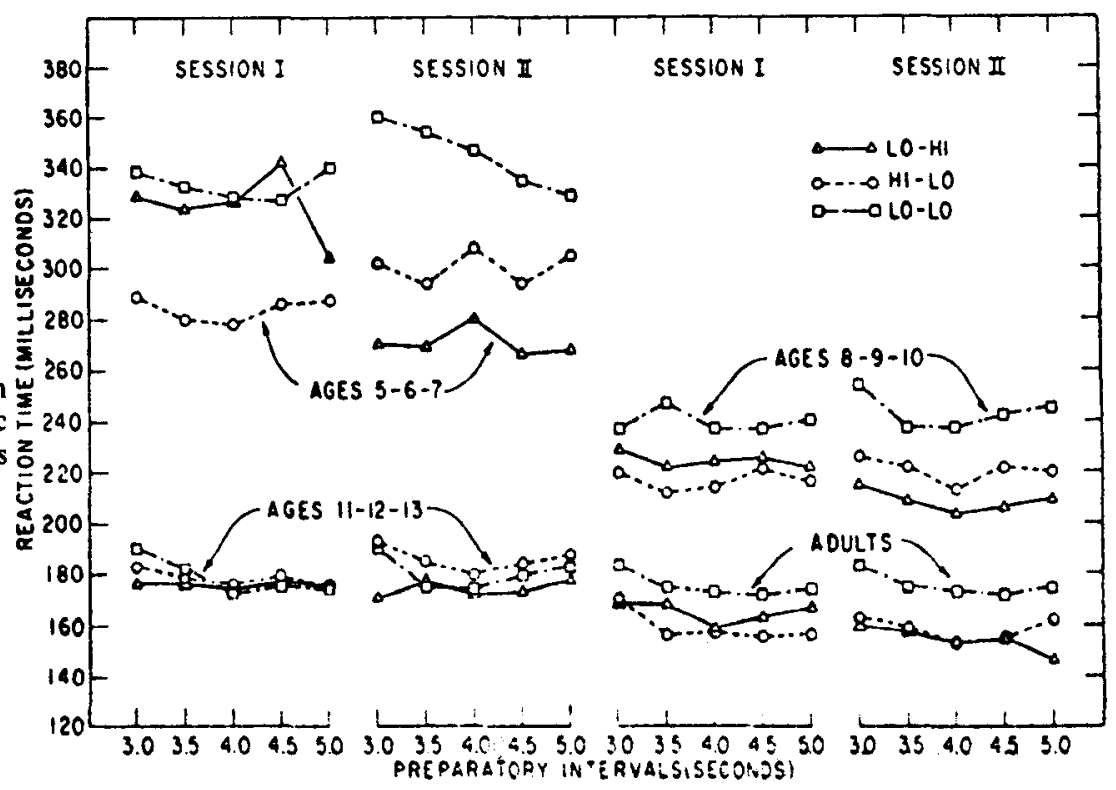


with those from the 1970 set were 47.5 and 29.2 , respectively, $\mathrm{df}=1 / 70, \mathrm{p}<.001$. Taking all $\mathrm{Pls}$ and all conditions into account, the average RT for the $2-\sec$ set was about $160 \mathrm{msec}$; for the 4 -sec set, $167 \mathrm{msec}$; and for the 1970 set, $198 \mathrm{msec}$.

In the present study the age differences in overall speed were, as is usual, large and highly reliable ( $\mathrm{F}$ ratios for the effect of age ranged from 27 to $47, \mathrm{df}=3 / 44$ ) in all comparisons, but with a single exception (low-low, 2-sec set second session: interaction of Age by PI significant at $p<.05$ ) they were not associated with any reliable differences in the shape of the RT by PI function in either series, in any condition, or in any session. It appears, in sum, that small-range PI sets of the sort used here, especially the 4-sec set, are not efficient for sensitive assessment of age differences in preparatory set as indexed by PI effects, in part because they fail to generate significant PI effects in the first place. We may, for this reason, assign to his fortuitous choice of a 4-sec set part of the author's (1964) failure to find a larger PI effect among kindergarten children than he found in adults.

The results from the 2-sec set are of interest when they are compared with the data of Jones (1937) and Goodenough (1935). Both of those investigators used short PIs in slightly irregular series, though they were not described precisely. If we take the data of the low-incentive Ss in the first session (those investigators did not use a high-incentive manipulation of the kind used here), the data points fall almost exactly on the curve drawn by Woodworth and Schlosberg (1954) from the Jones and Goodenough data, taking years $6,9,12$, and 21 as the appropriate chronological ages for comparison. We may assume from this that our Ss are not unrepresentative of their age groups.

A number of results in the present study, though incidental to the main question here, are of interest with respect to the earlier (1970) results concerning the effects of various incentive patterns. In the 1970 report the salient results were that the shift from low to high incentive had resulted in a large significant facilitation of performance, with the younger groups benefiting more than the older; that there had been a small facilitative practice effect in the low-low groups; and, most surprisingly, that there had been no decrement in the groups who were shifted from high to low incentive.

In the present experiment, $S$ by session analyses were again done. There was a small improvement with the shift from low to high incentive, but it was significant at the .05 level only among the nines in each PI set and nearly so among the sixes-a result generally like but much weaker than that of 1970 . In the low-low groups, no significant changes took place, so the facilitation that had been associated with practice in the earlier study did not appear in this one. And in the group going from high to low incentive, there was actual significant $(\mathrm{p}<.05)$ decrement in the present case among two of the age groups (the twelves and nines) in the $2 \cdot \sec$ set and a near-significant $(p<.06)$ decremental change among the sixes under the 4-sec set. This result again contrasts with the 1970 data, in which the high-low Ss showed no loss.

The three incentive patterns were ordered in the same way in terms of degree of facilitation in the second session (or half-session), but some decremental process not present in the 1970 data appears to have affected all of them. Since all the data were taken at one sitting, the most likely factor is habituation or fatigue, but nothing can be confidently said about that now because the change in method of data taking was confounded with the accompanying change in PI distribution.

\section{EXPERIMENT II}

Experiment II was done to test the hypothesis stated in the introduction: Namely, that for a given central value of PI, the effect of a set of PIs on RT would be a function of their range and that, for any given range, the effect would vary inversely with the size of the central value. In the comparisons made in Experiment I, differences in range were confounded with differences in central value. In the present experiment, values of the two dimensions are combined factorially, with every $S$ responding under every combination of values.

\section{Method}

The Ss were eight male graduate and undergraduate students. The apparatus was the same as that used in Experiment.I, except that a five-pole switch was used to put one of the five timers (controlling one of the five PIs) into the circuit on each trial and a screen shielded the Es' activities from the $S$.

The two central PI values were 6 and $12 \mathrm{sec}$, and the three ranges were 2, 6, and $10 \mathrm{sec}$. Each of the resulting six conditions can be conveniently identified by the numerical value of the central PI followed by that of the range, yielding 6-2, 6-6, 6-10, $12-2,12-6,12-10$. The ranges were symmetrical about the central values, so that the PIs of Condition $6-10$, for example, were $1,3.5,6,8.5$, and $11 \mathrm{sec}$.

For every condition there were two blocks of 26 trials, and within each block the PIs were arranged so that each was preceded by itself and all other PIs at least once. In each session, three conditions were run. Half ( 26 trials) of each of the three conditions was run in the first half of the session, and, following a brief rest, the other half was run, with the order of the conditions reversed to counterbalance for fatigue effects. In the next session, the other three conditions were run, so that each pair of sessions constituted a complete experiment. All Ss completed two such pairs of sessions, or four sessions in all.

Within a $S$, the order of administration of conditions in the first pair of sessions was changed in the second, and the orders were balanced across $S s$ within each pair of sessions. Each of two Es ran four of the $S s$ in the first pair of sessions and the other four in the last pair. The high-incentive condition was used throughout, consisting in this case of a chip worth 2 cents for every criterially fast reaction.

Data used in analysis were the median RTs for each PI of each condition. The first RT produced in each block of 26 trials was ignored, leaving five RTs for each PI. Two such blocks made up a condition, and the median, taken as the score halfway between the fifth and sixth largest of the $10 \mathrm{RT}$, was calculated for each PI. These medians-five for each condition for each $S$-were the scores used in analysis. 
Table 1

Mean of Individual Median RTs (Milliseconds) at Each PI in Each Condition

\begin{tabular}{|c|c|c|c|c|c|c|c|c|c|c|c|}
\hline \multirow{2}{*}{$\begin{array}{l}\text { Range } \\
\text { of } \mathrm{Pls}\end{array}$} & & \multicolumn{10}{|c|}{ Central PI } \\
\hline & & \multicolumn{5}{|c|}{$6 \mathrm{Sec}$} & \multicolumn{5}{|c|}{$12 \mathrm{Sec}$} \\
\hline $10 \mathrm{Sec}$ & $\begin{array}{l}\text { PI } \\
\text { RT }\end{array}$ & $\begin{array}{r}1 \\
195\end{array}$ & $172^{3.5}$ & $\begin{array}{r}6 \\
164\end{array}$ & $\begin{array}{c}8.5 \\
158\end{array}$ & $\begin{array}{r}11 \\
150\end{array}$ & $\begin{array}{r}7 \\
181\end{array}$ & $177^{9.5}$ & $\begin{array}{r}12 \\
172\end{array}$ & $\begin{array}{c}14.5 \\
169\end{array}$ & $\begin{array}{r}17 \\
160\end{array}$ \\
\hline $6 \mathrm{Sec}$ & $\begin{array}{l}\text { PI } \\
\text { RT }\end{array}$ & $\begin{array}{r}3 \\
176\end{array}$ & $\begin{array}{c}4.5 \\
152\end{array}$ & $\begin{array}{r}6 \\
158\end{array}$ & $\begin{array}{c}7.5 \\
159^{7 .}\end{array}$ & $\begin{array}{r}9 \\
149\end{array}$ & $\begin{array}{r}9 \\
183\end{array}$ & $\begin{array}{c}10.5 \\
176\end{array}$ & $\begin{array}{r}12 \\
173\end{array}$ & $\begin{array}{c}13.5 \\
171\end{array}$ & $\begin{array}{r}15 \\
165\end{array}$ \\
\hline $2 \mathrm{Sec}$ & $\begin{array}{l}\text { PI } \\
\text { RT } \\
\end{array}$ & $\begin{array}{r}5.0 \\
162 \\
\end{array}$ & $\begin{array}{c}5.5 \\
162 \\
\end{array}$ & $\begin{array}{r}6.0 \\
156 \\
\end{array}$ & $\begin{array}{c}6.5 \\
154 \\
\end{array}$ & $\begin{array}{c}7.0 \\
150 \\
\end{array}$ & $\begin{array}{r}11 \\
173 \\
\end{array}$ & $\begin{array}{c}11.5 \\
174\end{array}$ & $\begin{array}{r}12 \\
170 \\
\end{array}$ & $\begin{array}{c}12.5 \\
169\end{array}$ & $\begin{array}{r}13 \\
162 \\
\end{array}$ \\
\hline
\end{tabular}

There were some irregularities in the nunning of Ss in the first pair of sessions: Every $S$ ran in every condition, but three of them had 75 trials in one and only 25 in another. Therefore, only the data from the last pair of sessions was considered for analysis, with the first pair constituting simply a practice.

Analy'ses were all within-S analyses of variance. The major one was an extended ABC $S$ design in which the correlated factors were central value, range, and PI, and the error term for any factor or interaction was the $S$ interaction with that factor or interaction.

\section{Results and Discussion}

Table 1 shows the mean RTs for each PI in each condition. An analysis of variance of these data involving as factors the central PI value, the range of PIs, and the PIs within any combination of range and central value showed that $\mathrm{PI}(\mathrm{F}=37.10, \mathrm{df}=4 / 28, \mathrm{p}<.001)$, range $(\mathrm{F}=5.28, \quad \mathrm{df}=2 / 14, \mathrm{p}<.05)$, their interaction $(F=3.62, \mathrm{df}=8 / 56, \mathrm{p}<.01)$, and the interaction of $\mathrm{PI}$ with central value $(F=5.52, \mathrm{df}=4 / 28, p<.01)$ were significant.

The form of the overall PI effect was typical, with poor RTs to the shortest PI in a set and faster RTs the longer the PI. In subsidiary analyses of variance of repeated measures, the PI effect was significant at the .005 level or better in all conditions except 12-2, in which the effect did not attain the .05 level. That is the condition, by hypothesis, least likely to show any PI effect, since the error of estimate of the central value may approximate the range of the PIs.

The tests of the main hypothesis are the reliability of the interactions of PI with range and with central value, both of which were, as noted, significant; it is clear that the Pl effect was larger the larger the range and smaller the larger the central value. The main effect of range indicates that there were faster RTs on the average with the shorter range, but this effect, as well as the interaction of range with $\mathrm{PI}$, occurred primarily within the 6-sec central value. Subsidiary analyses of each central value separately showed that neither effect (range, or Range by PI interaction) obtained in the 12-sec value, while both did in the 6-sec value: in the latter, for the effect of range, $F$ was $7.1, \mathrm{df}=2 / 14$, $p<.01$; for the interaction of range with $\mathrm{PI}, \mathrm{F}=4.1$. $\mathrm{df}=8 / 56, \mathrm{p}<.001$. The overall effect of the difference in central value was only marginally significant $(\mathrm{p}<.10)$, with the RTs under the 12-sec value being generally slower. We may continue to assume, pending more reliable evidence, that the effect of central value and its interaction with range would be reliable if greater differences in central values had been employed.

\section{CONCLUDING REMARKS}

The original question was whether the effect of PI on RT varied as a function of the range of Pls, their central value, and the interaction of these effects. They do, and the disparity between the results of two of the author's studies of age differences in preparatory set effects is to some degree resolvable because they do: In one of the studies (1964), the range and central value used were not sufficient to produce the effects to any substantial degree in either age group; and in the other study (1970), they were.

In connection with age differences in preparatory set, it should be noted that the effects of increasing range while holding central value constant are to slow down RT overall and produce large PI effects, i.e., they are the same effects one observes with decreasing age, holding the PI distribution constant. Increasing the range, the task is made more difficult by increased requirements to maintain readiness to respond and increasing adaptation level effects, i.e., a greater load is put on one's attention. In decreasing age without changing the range, the load is the same, but the attention brought to bear on it probably decreases (for reasons discussed in the 1970 paper) so that the relative load also increases.

\section{REFERENCES}

Drazin. E. E. Effects of foreperiod, foreperiod variability, and probability of stimulus occurrence on simple reaction time. Journal of Experimental Psychology. 1961.62. 43-50.

Elliott. R. Physiological activity and performance: A comparison of kindergarten children with young adults. Psychological Monographs. 1964. 78, No. 10(Whole No. 587).

Elliott. $R$. Heart rate and reaction speed as functions of incentive magnitude and probability of success: $A$ replication and extension. Journal of Experimental Research in Personality. 1966. 1. 174-178.

Elliott. R. Simple reaction time: Effects associated with age. preparatory interval. incentive-shift and mode of presentation. Journal of Experimental Child Psychology. 1970. 9. 86-107.

Elljott. R. Simple reaction time in children: Effects of incentive. incentive-shift and other training variables. Jourmal of Experimental Child Psycholog!. 1972. 13. 540-557. 
Goodenough, F. L. The development of the reactive process from early childhood to maturity. Journal of Experimental Psychology, 1935, 18, 41-450.

Jones, H. E. Reaction time and motor development. American Journal of Psychology, 1937, 50, 181-194.

Karlin, L. Reaction time as a function of foreperiod duration and variability. Journal of Experimental Psychology. 1959. 58, 185-191.

Woodworth, R. S.. \& Schlosberg, H. E. Experimental psichology. New York: Henry Holt. 1954.

(Received August 9. 1972.) 